\title{
Parametrização das Equações de Hargreaves \& Samani e Ångström-Prescott Para Estimativa da Radiação Solar em Goiânia-GO
}

\author{
Elen Pereira de Paiva Bento da Silva ${ }^{1}$ (D), Camilla Nascimento Brito ${ }^{1}$ (D), \\ Adão Wagner Pêgo Evangelista ${ }^{2}$ (D), Derblai Casaroli ${ }^{2}$ \\ ${ }^{1}$ Programa de Pós-Graduação em Agronomia, Escola de Agronomia, Universidade Federal de \\ Goiás, Goiânia, GO, Brasil. \\ ${ }^{2}$ Setor de Engenharia de Biossistemas, Escola de Agronomia, Universidade Federal de Goiás, \\ Goiânia, GO, Brasil.
}

Recebido em: 12 de Abril de 2021 - Aceito em: 28 de Julho de 2021

\begin{abstract}
Resumo
A radiação solar é uma variável climatológica amplamente utilizada em estudos climáticos e pode ser mensurada por meio de estações meteorológicas. Devido ao alto custo e difícil manutenção dos equipamentos de mensuração, é possível estimá-la a partir de modelos matemáticos. Assim, o presente estudo objetivou calibrar e validar os parâmetros da equação de Ångström-Prescott e de Hargreaves \& Samani para realizar a estimativa da radiação solar incidente em Goiânia, Goiás. Dados históricos diários de temperatura máxima $\left(T_{\max } ;{ }^{\circ} \mathrm{C}\right)$ e mínima $\left(T_{\min } ;{ }^{\circ} \mathrm{C}\right)$, radiação solar incidente $\left(R s, \mathrm{MJ} \mathrm{m}^{-2}\right.$ dia $\left.^{-1}\right)$ e duração da insolação $\left(n, \mathrm{~h} \mathrm{dia}^{-1}\right)$ da região foram utilizados. Os parâmetros foram calibrados para períodos mensais e sazonais empregando o método dos mínimos quadrados. Os parâmetros estimados foram validados por meio do índice de concordância $(d)$, do coeficiente de correlação $(\mathrm{r})$ e da raiz do erro quadrático médio (RMSE). O parâmetro $k_{R s}$ variou de 0,12 a 0,14 enquanto o parâmetro $a$ variou entre 0,06 e 0,43 e o $b$ de 0,16 a 0,72 . As estimativas nos períodos sazonais apresentaram melhor desempenho quando comparadas aos mensais. Os índices obtidos demonstraram boas estimativas para os parâmetros nos períodos sazonais, sendo adequados para estimar a radiação solar no município de Goiânia.
\end{abstract}

Palavras-chave: energia disponível, coeficiente empírico, agrometeorologia.

\section{Parameterization of the Hargreaves \& Samani and Ångström-Prescott Equations for Estimating Solar Radiation in Goiânia-GO.}

\begin{abstract}
Solar radiation is a climatological variable widely used in climate studies and can be measured using meteorological stations. Due to the high cost and complex maintenance of measurement equipment, it is possible to estimate it using mathematical models. Thus, the present study aimed to calibrate and validate the parameters of the Ångström-Prescott and Hargreaves \& Samani equation to estimate the solar radiation incident in Goiânia, Goiás. Daily maximum temperature historical data $\left(T_{\max } ;{ }^{\circ} \mathrm{C}\right)$ and minimum $\left(T_{\min } ;{ }^{\circ} \mathrm{C}\right)$, incident solar radiation $\left(R s, \mathrm{MJ} \mathrm{m}^{-2} \mathrm{dia}^{-1}\right)$ and sunshine duration $\left(n, \mathrm{~h} \mathrm{dia}^{-1}\right)$ in the region were used. The parameters were calibrated for monthly and seasonal periods using the leastsquares method. The estimated parameters were validated using the Willmott index $(d)$, the correlation coefficient $(\mathrm{r})$, and the root of the mean square error $(R M S E)$. The parameter $k_{R s}$ varied from 0.12 to 0.14 , while the parameter $a$ ranged between 0.06 and 0.43 , and the $b$ varied from 0.16 to 0.72 . The estimates in the seasonal periods performed better when compared to the monthly ones. The obtained indices demonstrated satisfactory estimates for the seasonal periods' parameters, being adequate to estimate the solar radiation in the Goiânia.
\end{abstract}

Keywords: available energy, empirical coefficient, agrometeorology.

Autor de correspondência: Elen Pereira de Paiva Bento da Silva, elenpaiva@discente.ufg.br. 


\section{Introdução}

A radiação solar, essencial para a vida na Terra, é a principal fonte de energia para toda a vida terrestre. Esse elemento meteorológico é utilizado em diversos estudos agrícolas, como a determinação da evapotranspiração de referência diária estimada $\left(\mathrm{ET}_{0}\right)$ (Leite et al., 2020; Quej et al., 2016), modelagem de crescimento de plantas (Santos et al., 2017), produção agrícola (Teixeira et al., 2015) e mudanças climáticas (Borges, 2010). Existem duas maneiras de determinar a energia da radiação solar. A radiação extraterrestre $\left(R_{a}\right)$ representa a energia solar incidente sem considerar os efeitos da atmosfera; ou seja, é o potencial incidente de energia da região. A radiação extraterrestre é calculada com base nos fatores que afetam o ângulo dos raios solares: latitude e dia juliano. Em condições normais, em que a atmosfera atua, a fração da energia solar que atinge a atmosfera terrestre, denominada radiação solar incidente, é menor que a radiação extraterrestre (Allen et al., 1998). Essa fração pode ser medida com radiômetros específicos, os piranômetros.

No entanto, a radiação solar incidente não é medida na maioria das estações meteorológicas do Brasil devido ao alto custo dos instrumentos e de manutenção, que exige calibração e limpeza contínua. Diante disso, a disponibilidade de dados é escassa para uso em vários estudos agrícolas. Como alternativa para este obstáculo, são comumente utilizados modelos matemáticos para estimar a radiação solar incidente (Babikir et al., 2018) que apresentam estimativas confiáveis. O método proposto por Ångström-Prescott tornou-se o mais utilizado. Em primeiro lugar, este modelo foi baseado na correlação linear entre a radiação solar incidente e a radiação solar extraterrestre e a razão entre a luz solar diária e o fotoperíodo (Ångström, 1924). Posteriormente, Prescott (1940) complementou a equação para que o parâmetro linear $(a)$ e o parâmetro angular $(b)$ pudessem ser ajustados estatisticamente (Jerszurki e Souza, 2013).

Outro modelo de estimativa da radiação solar incidente, que se destaca, é o proposto por Hargreaves e Samani (1982). Este método é baseado na amplitude térmica, radiação solar extraterrestre $\left(R_{a}\right)$ e no parâmetro $k_{R s}$ (Leite et al., 2020). Nesse modelo, a amplitude térmica diária indica a radiação solar incidente (Jerszurki e Souza, 2013).

Alguns coeficientes foram propostos para ambos os modelos (Allen et al., 1998). Para o método ÅngströmPrescott, foram sugeridos 0,25 e 0,50 para os parâmetros linear e angular, respectivamente. E para o método Hargreaves e Samani, foi apresentado 0,16 para regiões do interior, como é o caso de Goiânia, Goiás, onde grandes corpos d'água não influenciam fortemente as massas de ar. No entanto, os parâmetros podem ser estimados com base nos dados de cada região para obter valores mais confiáveis para estimar a radiação solar incidente. Assim, o presente estudo teve como objetivo calibrar e validar os parâmetros das equações de Ångström-Prescott $(a$ e $b) \mathrm{e}$ Hargreaves \& Samani $\left(k_{R s}\right)$ para estimar a radiação solar incidente em Goiânia, Goiás.

\section{Material e Métodos}

Os dados climáticos, utilizados no presente estudo (fevereiro de 2014 a janeiro de 2020), foram medidos nas estações convencionais e automáticas da Universidade Federal de Goiás, Goiânia (16 $35^{\prime} 52.72648^{\prime \prime}$ S; 49 16'39.84946" O e altitude de $729.846 \mathrm{~m}$ ). A região possui classificação climática do tipo Aw, que indica um clima tropical úmido-seco caracterizado por duas estações bem definidas: uma chuvosa no verão e outra seca no inverno (Köppen \& Geiger, 1928). Os dados históricos diários usados foram temperatura máxima e mínima do ar $\left({ }^{\circ} \mathrm{C}\right)$, radiação solar incidente acumulada $\left(R s, \mathrm{MJ} \mathrm{m}^{-2}\right.$ dia $\left.^{-1}\right)$ e insolação $\left(n, \mathrm{~h} \mathrm{dia}^{-1}\right)$, de fevereiro de 2014 a janeiro de 2020.

Para a calibração, os parâmetros lineares $(a)$ e angulares $(b)$ foram estimados empregando a equação de Ångström-Prescott (Ångström, 1924; Prescott, 1940) (Eq. (1)), e o coeficiente empírico $\left(k_{R s}\right)$ foi calculado usando a equação de Hargreaves e Samani (Hargreaves e Samani, 1982) (Eq. (2)).

$$
\begin{gathered}
R_{s}=R_{a} \cdot\left[a+b \cdot\left(\frac{n}{N}\right)\right] \\
R_{s}=k_{R s} \cdot\left(T_{\max }-T_{\min }\right)^{0,5} \cdot R_{a}
\end{gathered}
$$

em que $R_{s}$ é a radiação solar incidente à superfície terrestre $\left(\mathrm{MJ} \mathrm{m}^{-2} \mathrm{dia}^{-1}\right) ; R_{a}$ é a radiação solar no topo da atmosfera $\left(\mathrm{MJ} \mathrm{m}^{-2} \mathrm{dia}^{-1}\right) ; a$ é o parâmetro linear (adimensional); $b$ é o parâmetro angular (adimensional); $n$ é a insolação diária (h); $N$ é o fotoperíodo calculado de acordo com a latitude local e dia juliano; $k_{R s}$ é o parâmetro empírico (adimensional); $T_{\max }$ e $T_{\min }$, são as temperaturas máximas e mínimas do ar $\left({ }^{\circ} \mathrm{C}\right)$, respectivamente. A radiação solar no topo da atmosfera $\left(R_{a}\right)$ foi calculada de acordo com Allen et al. (1998).

Os parâmetros foram estimados para períodos mensais e sazonais. Para tanto, foram selecionados aleatoriamente cinco anos: 2014, 2016, 2017, 2018 e 2020. Os parâmetros foram estimados aplicando o método dos mínimos quadrados sobre as estimativas da radiação solar incidente calculados a princípio com os parâmetros da literatura. Para isso, foi utilizado o recurso solver do Excel. Em seguida, traçou-se a regressão linear entre a $R_{S}$ estimada e a $R_{s}$ obtida.

Após a calibração, os anos restantes foram utilizados para que o método de estimativa fosse validado. Primeiramente, a $R_{s}$ estimada foi calculada empregando os parâmetros obtidos na calibração. Após, foi calculado o índice de concordância (d) de acordo com Willmott et al. (1985) (Eq. (3)), o coeficiente de correlação (r) e a raiz do erro 
quadrático médio (RMSE) (Eq. (4)). Também foi traçada a regressão linear entre a $R_{S}$ estimada e a $R_{S}$ obtida.

$$
\begin{gathered}
d=1-\left[\frac{\sum\left(R_{s e}-R_{s o}\right)^{2}}{\sum\left(\left|R_{s e}-\overline{R_{s o}}\right|+\left|R_{s o}-\overline{R_{s o}}\right|\right) 2}\right] \\
R M S E=\sqrt{\frac{\sum\left(R_{s e}-R_{s o}\right)^{2}}{n}}
\end{gathered}
$$

em que $d$ é o índice de concordância; RMSE é o raiz do erro quadrático médio; $R_{s e}$ é a radiação solar incidente estimada ( $\left(\mathrm{MJ} \mathrm{m}^{-2} \operatorname{dia}^{-1}\right) ; R_{s o}$ é a radiação solar incidente obtida (MJ m$\left.{ }^{-2} \mathrm{dia}^{-1}\right) ; n$ é o tamanho da amostra.

\section{Resultados e Discussão}

\subsection{Calibração}

Na Fig. 1 encontra-se os ajustes dos parâmetros, por meio da regressão linear entre a $R_{s}$ estimada e a $R_{S}$ obtida. As Figs. 1A e 1B mostram a regressão linear para os pe- ríodos mensais e sazonais, respectivamente, da equação de Ångström-Prescott. O ajuste dos valores calibrados foi satisfatório, considerando o alto valor do coeficiente de determinação $\left(R^{2}\right)$ obtido nas duas análises $\left(R^{2}=0,8920\right.$; $\mathrm{R}^{2}=0,8535$ ).

As Figs. 1C e 1D mostram a regressão linear para os períodos mensais e sazonais, respectivamente, da equação de Hargreaves \& Samani. O ajuste dos valores calibrados para o parâmetro $k_{R s}$ também foi satisfatório visto que o coeficiente de determinação também obteve altos valores. No entanto, percebe-se que o $\mathrm{R}^{2}$ para os períodos sazonais é pouco mais elevado $\left(\mathrm{R}^{2}=0,8924 ; \mathrm{R}^{2}=0,9117\right)$.

Os parâmetros estimados das equações de Hargreaves e Samani e Ångström-Prescott, ajustados para períodos mensais e sazonais, estão apresentados na Tabela 1. Nos períodos mensais, os valores ajustados para o parâmetro linear $(a)$ variaram de 0,20 (dezembro) a 0,43 (maio) e para o parâmetro angular $(b)$ variaram de 0,16 (dezembro) a 0,66 (maio). Para o parâmetro $k_{R s}$, não houve variações muito altas. No entanto, o valor mais elevado foi para abril $(0,14)$, sendo os meses de junho, julho e agosto o menor valor $(0,12)$. Considerando os períodos sazonais, os valores do parâmetro linear $(a)$ variaram de
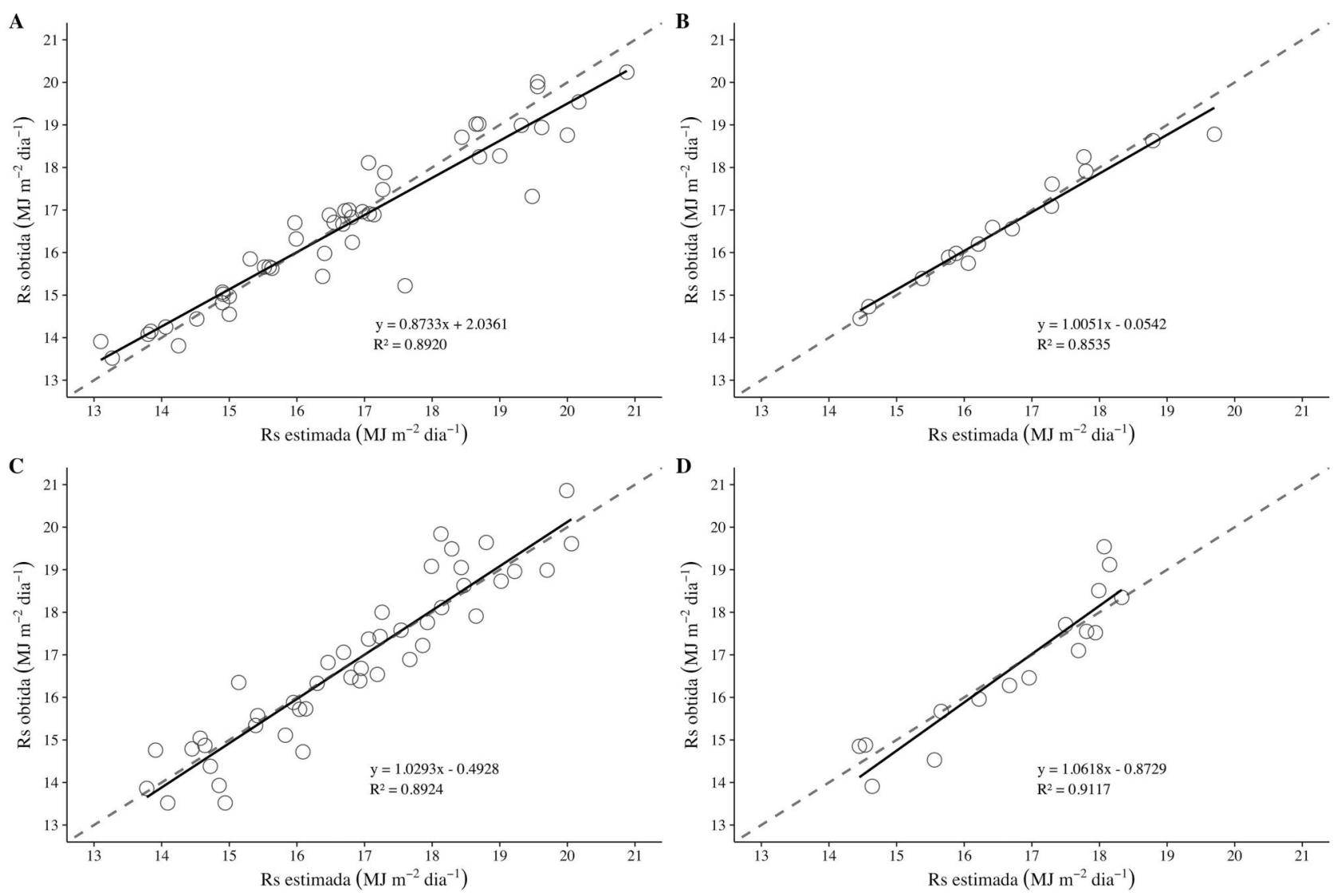

Figura 1 - Regressão linear entre a radiação solar incidente estimada $\left(R_{s e}\right)$ e a radiação solar incidente obtida $\left(R_{s o}\right)$ usando os modelos de Hargreaves e Samani e Ångström-Prescott para calibrar os parâmetros das equações em Goiânia, Goiás, Brasil. A linha tracejada indica a reta 1:1. (A) Calibração dos parâmetros $a$ e $b$ para períodos mensais. (B) Calibração dos parâmetros $a$ e $b$ para períodos sazonais. (C) Calibração do parâmetro $k_{R s}$ para períodos mensais. (D) Calibração do parâmetro $k_{R s}$ para períodos sazonais. 
Tabela 1 - Valores estimados do parâmetro linear (a), parâmetro angular (b) e parâmetro $k_{R s}$ ajustados para períodos mensais e sazonais em Goiânia, Goiás, Brasil.

\begin{tabular}{lccccc}
\hline Períodos & $a$ & $b$ & $\mathrm{R}^{2}$ & $k_{R s}$ & $\mathrm{R}^{2}$ \\
\hline Janeiro & 0,27 & 0,51 & 0,8708 & 0,13 & 0,9677 \\
Fevereiro & 0,35 & 0,34 & 0,8491 & 0,13 & 0,6803 \\
Março & 0,36 & 0,21 & 0,9437 & 0,13 & 0,3368 \\
Abril & 0,26 & 0,43 & 0,6376 & 0,14 & 0,6038 \\
Maio & 0,43 & 0,16 & 0,9528 & 0,13 & 0,3290 \\
Junho & 0,14 & 0,54 & 0,2079 & 0,12 & 0,1271 \\
Julho & 0,06 & 0,59 & 0,9980 & 0,12 & 0,9065 \\
Agosto & 0,25 & 0,40 & 0,7401 & 0,12 & 0,9476 \\
Setembro & 034 & 0,29 & 0,7420 & 0,13 & 0,1038 \\
Outubro & 0,33 & 0,30 & 0,9625 & 0,13 & 0,8966 \\
Novembro & 0,29 & 0,45 & 0,9460 & 0,13 & 0,9948 \\
Dezembro & 0,20 & 0,66 & 0,7949 & 0,13 & 0,4475 \\
Primavera & 0,29 & 0,43 & 0,9282 & 0,13 & 0,3357 \\
Verão & 0,32 & 0,36 & 0,8991 & 0,13 & 0,6386 \\
Outono & 0,06 & 0,72 & 0,9942 & 0,13 & 0,4844 \\
Inverno & 0,19 & 0,47 & 0,9903 & 0,13 & 0,9903 \\
\hline
\end{tabular}

0,06 (outono) a 0,32 (verão) e do parâmetro angular (b) variaram de 0,36 (verão) a 0,72 (outono). Os valores do parâmetro $k_{R s}$ foram os mesmos $(0,13)$ para os períodos sazonais.

O parâmetro da equação de Hargreaves \& Samani obteve baixo coeficiente de determinação para vários períodos: março $(0,3368)$, maio $(0,3290)$, abril $(0,1271)$, setembro $(0,1038)$, dezembro $(0,4475)$, primavera $(0,3357)$ e outono $(0,4844)$. Os coeficientes de determinação variaram de 0,6376 (abril) a $(0,9980)$ julho para a equação de Ångström-Prescott. Esses valores apresentam alta associação devido à proximidade de 1 . Porém, para junho, o coeficiente de determinação foi de 0,2079 . O baixo desempenho nesses períodos, tanto no modelo de Hargreaves \& Samani quanto Ångström-Prescott, é atribuído ao baixo volume e à qualidade dos dados disponíveis para a região (fevereiro de 2014 a janeiro de 2020), bem como no estudo de Macapá-AP produzido por Belúcio et al. (2014).

Neste estudo, observa-se que os valores dos parâmetros linear $(a)$ e angular (b) ajustados diferiram dos recomendados por Allen et al. (1998), que, respectivamente, são 0,25 e 0,50 , evidenciando a importância da calibração local. Outros estudos também encontraram parâmetros que diferem do recomendado. Carvalho et al. (2011) encontraram, para a região de Seropédica - RJ, valor médio de 0,273 para $a$ e 0,457 para $b$.

Para a região de Caicó - RN, Medeiros et al. (2017) encontraram um valor médio de 0,36 para $a$ e 0,38 para $b$. Esses valores mostram que Caicó - RN apresentou alto valor de $a$ por ser uma região de clima semiárido com nuvens menos espessas. $\mathrm{O}$ clima da região proporciona maior transmissividade da atmosfera e menor atenuação da radiação solar em relação à Seropédica - RJ. O inverso ocorre com valores $b$ que diminuem com o aumento de $a$. Segundo Hinrichsen (1994), o coeficiente $a$ representa fisicamente a fração da radiação difusa, enquanto $b$ quantifica a fração da radiação direta. Os resultados obtidos corroboram com os de outros autores como Asilevi et al. (2019) e Martim et al. (2020) que encontraram maior transmissividade devido as regiões de estudo apresentarem baixa precipitação e baixa presença de nuvens, resultado em maiores valores do parâmetro $a$.

É impossível pré-definir como será a dinâmica de variação dos parâmetros linear e angular ao longo do ano. Assim, é essencial a parametrização para cada região específica, uma vez que os parâmetros são condicionados por dados particulares como latitude, altitude, continentalidade e região (Lena et al., 2020). Buriol et al. (2012) também constataram que o parâmetro linear foi menor no mês de julho para o município de Santa Maria - RS. Isso indica maior transmissividade da atmosfera neste período, uma vez que o parâmetro linear está relacionado à radiação solar difusa.

Os valores do coeficiente $k_{R s}$ ajustado também diferiram do recomendado pela FAO $(0,16)$, mostrando que a utilização desse valor superestimou a radiação solar incidente em Goiânia. Alguns autores também encontraram valores de $k_{R S}$ diferentes do usual. Para Telêmaco BorbaPR (classificação de Köppen $=\mathrm{Cfa}$ ), foi encontrado valor para $k_{R s}$ igual a 0,12 (Jerszurki e Souza, 2013). Para a região sul da Argélia (classificação de Köppen $=$ Csa), Ihaddadene et al. (2019) encontraram valores entre 0,143 e 0,146 . Pode-se observar que essas regiões são locais com clima mais ameno e subtropical, com baixa amplitude térmica, segundo a classificação climática de Köppen.

A equação de Hargreaves \& Samani considera que a amplitude térmica é um indicador da fração da energia solar que atinge a superfície terrestre (Allen et al., 1998). Assim, esses valores indicaram uma superestimação da radiação solar incidente quando o coeficiente proposto por Allen et al. (1998) é usado. Porém, para o interior de Alagoas (classificação de Köppen = BSh), os estudos encontraram valores entre 0,168 e 0,179 (Lyra et al., 2015), superiores ao usual. De acordo com sua classificação climática, este alto valor para o parâmetro $k_{R s}$ pode ser atribuído às altas temperaturas que a região apresenta. Assim, o valor usual $(0,16)$ subestima a radiação solar que atinge a região.

Junho, julho e agosto são os meses mais frios do ano na região de Goiânia. Isso contribui para o aumento da amplitude térmica que é considerada neste modelo. Consequentemente, o parâmetro $k_{R s}$ deve ser menor para manter a identidade da equação (Lyra et al., 2015). Esses são os meses com a menor duração do dia devido ao solstício de inverno no hemisfério sul (Hartmann, 1994). Esse fenômeno implica no número de horas de luz solar que a 
região receberá, influenciando diretamente na radiação solar incidente. Embora a insolação em regiões tropicais não varie muito, notou-se que a curta variação influenciou na radiação solar incidente nos meses de junho a agosto.

Em contraste, a proximidade de abril com o fim do período de chuva e a elevada temperatura ainda característica dos meses de verão caracteriza o alto valor encontrado para o parâmetro $k_{R s}$. Estes fatores contribuem com a maior incidência de radiação solar direta. Como resultado, a amplitude térmica pode diminuir. Sobre as estações do ano, observa-se que o valor do parâmetro $k_{R s}$ também difere do proposto por Allen et al. (1998), mas não variou entre as estações.

\subsection{Validação}

As Figs. 2A e 2B mostram as regressões lineares da $R_{s}$ obtida na estação meteorológica e da $R_{s}$ estimada empregando os valores dos parâmetros $a$ e $b$ nos anos utilizados para validação. A validação do ajuste mensal obteve alto coeficiente de determinação $\left(\mathrm{R}^{2}=0,8038\right)$; alto coeficiente de correlação $(\mathrm{r}=0,8966)$, elevado índice de Willmott $(d=0,7221)$ e $R M S E$ igual a $1,1285 \mathrm{MJ} \mathrm{m}^{-2} \mathrm{dia}^{-1}$
(Fig. 2A). A validação do ajuste sazonal também obteve alto coeficiente de determinação $\left(\mathrm{R}^{2}=0,8591\right)$; alto coeficiente de correlação $(\mathrm{r}=0,9269)$, elevado índice de Willmott $(d=0,7358)$ e $R M S E$ igual a $0,8424 \mathrm{MJ} \mathrm{m}^{-2} \mathrm{dia}^{-1}$ (Fig. 2B).

As regressões lineares entre a $R_{S}$ obtida na estação meteorológica e da $R_{S}$ estimada empregando os valores de $k_{R s}$. estimados estão ilustradas nas Figs. $2 \mathrm{C}$ e $2 \mathrm{D}$. A Fig. 2C mostra o ajuste para períodos mensais. A validação do ajuste mensal obteve alto coeficiente de determinação $\left(\mathrm{R}^{2}=0,8897\right)$; alto coeficiente de correlação $(\mathrm{r}=0,9432)$, elevado índice de Willmott $(d=0,7028)$ e RMSE igual a $0,8342 \mathrm{MJ} \mathrm{m}^{-2} \mathrm{dia}^{-1}$. A Fig. 2D mostra o ajuste para períodos sazonais. A validação do ajuste sazonal também obteve alto coeficiente de determinação $\left(\mathrm{R}^{2}=0,8866\right)$; alto coeficiente de correlação $(\mathrm{r}=0,9416)$, elevado índice de Willmott $(d=0,7124)$ e RMSE igual a $0,4498 \mathrm{MJ} \mathrm{m}^{-2} \mathrm{dia}^{-1}$.

Os elevados valores do coeficiente de determinação $\left(\mathrm{R}^{2}\right)$ e correlação $(\mathrm{r})$ indicam que as estimativas foram precisas e possuem correlação com os dados obtidos na estação meteorológica. O índice de concordância de Willmott demonstra alta acurácia na validação dos parâmetros
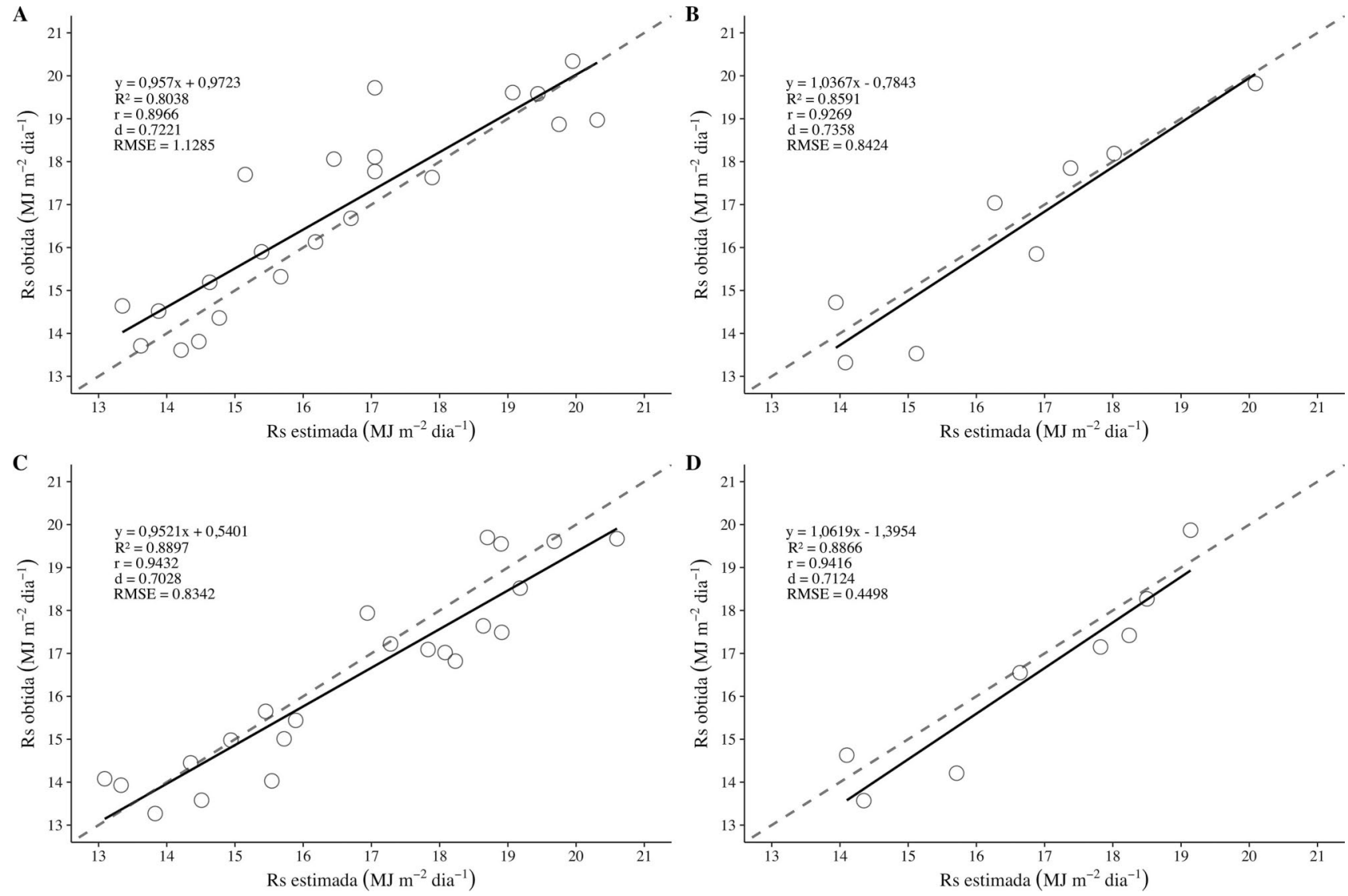

Figura 2 - Regressão linear entre radiação solar incidente estimada $\left(R_{s e}\right)$ e radiação solar incidente obtida $\left(R_{s o}\right)$ usando os modelos de Hargreaves \& Samani e Ångström-Prescott para calibrar os parâmetros das equações em Goiânia, Goiás, Brasil. A linha tracejada indica a reta 1:1. (A) Regressão linear dos períodos mensais para validação dos parâmetros $a$ e $b$. (B) Regressão linear dos períodos sazonais para validação dos parâmetros $a$ e $b$. (C) Regressão linear dos períodos mensais para validação do parâmetro $k_{R s}$. (D) Regressão linear dos períodos sazonais para validação do parâmetro $k_{R s}$. 
dos modelos, tanto nos períodos mensais quanto nos períodos sazonais. Nota-se que os valores de RMSE foram baixos, o que indica variação baixa no erro da estimativa.

\section{Conclusão}

Os coeficientes estimados neste estudo diferem dos propostos por Allen et al. (1998), que superestimam a radiação solar para a região em estudo. Os coeficientes encontrados no presente trabalho, tanto para a equação de Hargreaves e Samani quanto para a equação de ÅngströmPrescott, podem ser usados para estimar a radiação solar para o município de Goiânia, Goiás, Brasil. No entanto, recomenda-se a utilização dos parâmetros sazonais por terem apresentado os melhores resultados tanto na calibração quanto na validação da estimativa. Além disso, recomendamos a realização de novas estimativas a partir de uma série de dados maior, tendo em vista os períodos que obtiveram baixos valores para o coeficiente de determinação.

\section{Referências}

ALLEN, R.G.; PEREIRA, L.S.; RAES, D.; SMITH, M. Crop Evapotranspiration: Guidelines for Computing Crop Water Requirements. Rome: United Nation FAO, paper $\mathrm{n}^{\circ} 56,1998$.

ÅNGSTRÖM, A. Solar, and terrestrial radiation. Quarterly Journal of the Royal Meteorological Society, v. 50, p. 121-1216, 1924.

BABIKIR, M.H.; NJOMO, D.; KHAYAL, M.Y.; TEMENE, H.D.; JOEL, D.T. Estimation of Direct Solar Radiation of Chad. Energy and Power Engineering, v. 10, p. 212-225, 2018.

BELÚCIO, L.P.; SILVA, A.P.N.; SOUZA, L.R..; MOURA, G.B.A. Radiação solar global estimada a partir da insolação para Macapá (AP). Revista Brasileira de Meteorologia, v. 29, n. 4, p. 494-504, 2014.

BORGES, V.P.; OLIVEIRA, A.S.; COELHO FILHO, M.A.; SILVA, T.S.M.; PAMPONET, B.M. Avaliação de modelos de estimativa da radiação solar incidente em Cruz das Almas, Bahia. Revista Brasileira de Engenharia Agrícola e Ambiental, v. 14, n. 1, p. 74-80, 2010.

BURIOL, G.A.; ESTEFANEL, V.; HELDWEIN, A.B; PRESTES, S.D.; HORN, J.F.C. Estimativa da radiação solar global a partir dos dados de insolação, para Santa Maria RS. Ciência Rural, v. 42, n. 9, p. 1563-1567, 2012.

CARVALHO D.F.; SILVA, D.G.; SOUZA, A.P.; GOMES, D.P.; ROCHA, H.S. Coeficientes da equação de Ångström-Prescott e sua influência na evapotranspiração de referência em Seropédica, RJ. Revista Brasileira de Engenharia Agrícola e Ambiental, v. 15, n. 8, p. 838-844, 2011.

HARGREAVES, G.H.; SAMANI, Z.A. Estimating potential evapotranspiration. Journal of Irrigation and Drainage Engineering, v. 108, p. 223-230, 1982.

HARTMANN, D. L. Global Physical Climatology. Amsterdam: Elsevier Inc., p. 498, 2016.
HINRICHSEN, K. The Ångström formula with coefficients having a physical meaning. Solar Energy, v. 52, n. 6, p. 491495, 1994

IHADDADENE, R.; IHADDADENE, N.; JED, M.E.H.O.A.B.; SOUZA, A. Daily global solar radiation estimation based on air temperature: case of study south of Algeria. E3S Web of Conferences, v. 80, n. 01002, p. 1-5, 2019.

JERSZURKI, D.; SOUZA, J.L.M. Parametrização das equações de Hargreaves \& Samani e Ångström-Prescott para estimativa da radiação solar na região de Telêmaco Borba, Estado do Paraná. Ciência Rural, v. 43, n. 3, p. 383-389, 2013.

LEITE, C.V.; CASAROLI, D.; VICENTE, M.R.; CESSA, R.M.A.; ALVES-JUNIOR, J. Reference evapotranspiration through Hargreaves method using the solar radiation estimation for Goiás State, Brazil. Revista Engenharia na Agricultura, v. 28, p. 274-292, 2020.

LENA, B.P.; JADOSKI, S.O.; JADOSKI, C.J.; RIGO, J.F. Coeficientes da equação de Ångström-Prescott para a região de Guarapuava-PR. Acta Iguazu, v. 9, n. 1, p. 123-136, 2020.

LYRA, G.B.; ZANETTI, S.S.; SANTOS, A.A.R.; SOUZA, J.L.; LYRA, G.B.; OLIVEIRA-JÚNIOR, J.F.; LEMES, M.A.M. Estimation of monthly global solar irradiation using the Hargreaves-Samani model and an artificial neural network for the state of Alagoas in northeastern Brazil. Theoretical and Applied Climatology, v. 125, p. 743-756, 2016.

MARTIM, C.C.; NOGUEIRA, J.S.; GUIA, L.E.B.; CURADO, L.F.A. Estimate of global radiation by the Ångström-Prescott model for Tocantins. Research, Society and Development, v. 9, n. 7, p. 1-13, e549974139, 2020.

MEDEIROS, F.J.; SILVA, C.M.S.; BEZERRA, B.G. Calibration of Ångström-Prescott equation to estimate daily solar radiation on Rio Grande do Norte State, Brazil. Revista Brasileira de Meteorologia, v. 32, n. 3, p. 409-416, 2017.

PRESCOTT, J.A. Evaporation from a water surface about solar radiation. Transactions of the Royal Society Science Australian, v. 64, p. 114-118, 1940.

QUEJ, V.H.; ALMOROX, J.; IBRAKHIMOV, M.; SAITO, L. Empirical models for estimating daily global solar radiation in Yucatán Peninsula, Mexico. Energy Conversion and Management, v. 110, p. 448-456, 2016.

SANTOS, M.P.; ZANON, A.J.; CUADRA, S.V.; STEINMETZ, $\mathrm{S}$; CASTRO, J.R.; et al. Yield and morphophysiological indices of irrigated rice genotypes in contrasting ecosystems. Pesquisa Agropecuária Tropical, v. 47, n. 3, p. 253264, 2017.

TEIXEIRA, G.C.S.; STONE, L.F.; HEINEMANN, A.B. Eficiência do uso da radiação solar e índices morfofisiológicos em cultivares de feijoeiro. Pesquisa Agropecuária Tropical, v. 45, n. 1, p. 9-17, 2015.

WILLMOTT, C.J.; ROWE, C.M.; MINTZ, Y. Climatology of the terrestrial seasonal water cycle. International Journal of Climatology, v. 5, p. 589-606, 1985.

License information: This is an open-access article distributed under the terms of the Creative Commons Attribution License (type CC-BY), which permits unrestricted use, distribution and reproduction in any medium, provided the original article is properly cited. 